\title{
Georges Offenstadt (1944-2019) \\ Un humaniste sans préjugé au service de la réanimation
}

\author{
In Memoriam Georges Offenstadt (1944-2019)
}

\author{
E. Maury \\ C SRLF et Lavoisier SAS 2019
}

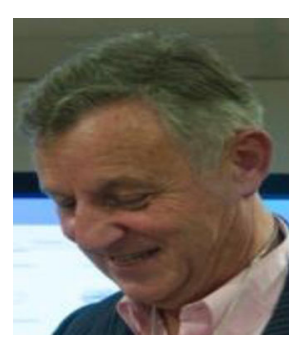

Georges Offenstadt nous a brutalement quittés le 9 avril dernier alors qu'il voyageait, seul comme à son habitude, et parcourait la Russie, pays d'origine de sa mère.

Il est difficile d'évoquer simplement Georges, tant il était actif (trop diraient certains), mais aussi entier et convivial tout en étant empreint d'humilité et de discrétion. Malgré ma tristesse, il m'est difficile de ne pas sourire quand je pense à lui, car son leitmotiv était : «Allez les potes !! Du tonus !! ».

Georges avait une personnalité qu'on pouvait ne pas apprécier mais qui ne laissait jamais indifférent. Sous des premiers contacts parfois déroutants perçait toujours la volonté de rendre service et surtout de bien faire. Il aidait les autres sans arrière-pensée, sans attendre quoi que ce soit en retour. Il a suivi avec discrétion les évolutions et carrières de plus jeunes collègues qu'il avait conseillés. Ceux qui l'ont connu et apprécié évoquent sa tendresse un peu rude ou sa bienveillance rugueuse. Jeune interne dans le service de réanimation de Saint-Antoine, j'ai d'abord été dérouté par son caractère entier et sa capacité à ne jamais être satisfait. Mais en l'observant, j'ai fini par comprendre qu'il était exigeant avec ses collaborateurs, mais pas plus qu'il ne l'était avec lui-même, et que la seule chose importante à ses yeux en tant que médecin était la qualité des soins apportés aux malades. J'ai le souvenir très vif de Georges alors qu'il était professeur corrigeant les pancartes, car elles étaient mal annotées, accélérant la réalisation de prescriptions qu'il jugeait essentielles ou brancardant un malade vers le bloc opératoire, car il fallait rapidement l'opérer. Il a été pour beaucoup un men-

\section{E. Maury $(\bowtie)$}

Hôpital Saint-Antoine, 184, rue du Faubourg-Saint-Antoine,

F-75012 Paris, France

e-mail : eric.maury@sat.aphp.fr tor, mais aussi et surtout un exemple, tant son investissement était total. Il fallait aller à l'essentiel mais sans pour autant rien négliger. Avec Georges, soigner signifiait prendre les malades à bras-le-corps. Au cours de la visite quotidienne, sa visite, rien n'était laissé au hasard ni à l'improvisation. Il nous a inculqué le souci d'anticiper les complications, car comme il aimait à le répéter « il fallait toujours avoir un coup d'avance ». Puis quand les problèmes avaient été réglés, on pouvait être convoqués (on était convoqués au début puis conviés, ensuite quand il apprenait à nous connaître) dans son bureau (sa caverne) pour discuter de façon dépassionnée (Fig. 1).

Je ne recevrai plus ses appels téléphoniques impromptus dans lesquels j'avais toujours droit à un tonitruant : " Maury qu'est-ce que tu fais? Passe me voir dans mon bureau, j'ai quelque chose pour toi ». Car il avait toujours une petite attention pour les membres de son équipe. Et ces moments passés ensemble étaient toujours l'occasion de partager une

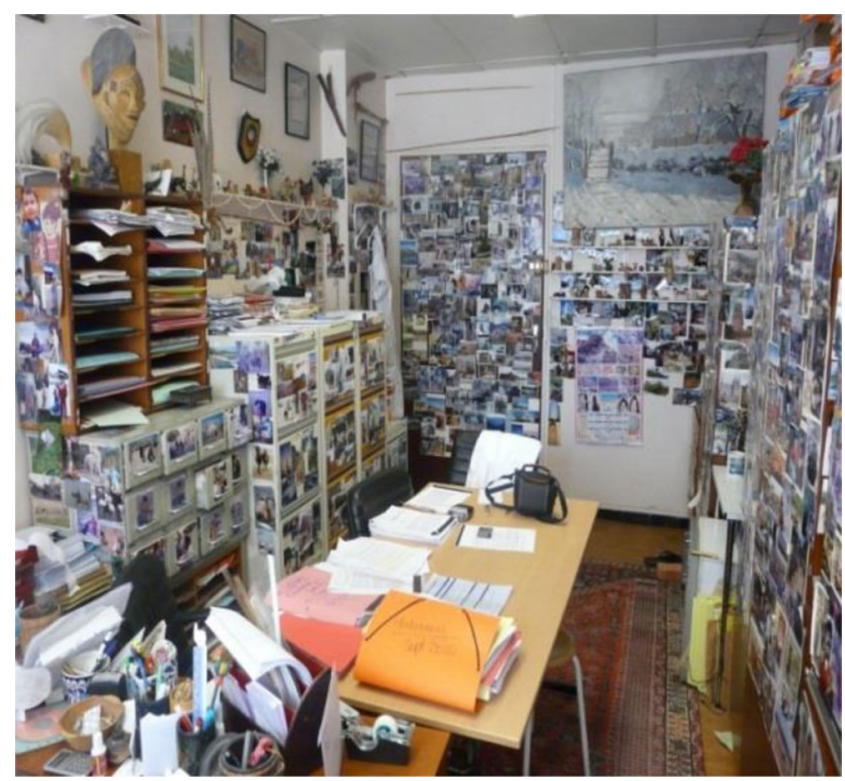

Fig. 1 Le bureau 
discussion, un gâteau, car nous étions tous les deux gourmands, ou un Coca (Light ou Zéro bien sûr). Il aura été un médecin dévoué, un défenseur de la discipline convaincu et un voyageur infatigable. Georges était attaché aux personnes et a toujours été persuadé que la qualité du service ne reposait que sur la qualité des hommes et des femmes qui y travaillaient et non sur le standing des locaux ou sur l'abondance et la modernité de l'équipement. La disparition prématurée de ses collaborateurs de toujours, Thierry Vassal puis Philippe Héricord, l'avait beaucoup attristé.

\section{Médecin réanimateur}

Son intérêt le porte initialement vers la cardiologie, puis rapidement vers la réanimation médicale dans laquelle s'exprimeront toujours sa soif de comprendre et son besoin d'efficacité. Il est nommé, en 1973, chef de clinique dans le tout nouveau service de réanimation médicale de l'hôpital SaintAntoine, hôpital auquel il restera très attaché, y devenant assistant, professeur puis chef de service. Son passage à l'hôpital Claude-Bernard avait suscité et développé chez lui un intérêt authentique pour les maladies infectieuses et les antibiotiques qu'il gardera tout au long de sa carrière. Il s'est aussi intéressé (je devrais plutôt dire passionné ou investi, car il accomplissait toujours ce qu'il entreprenait en profondeur) à l'hygiène, aux troubles hydroélectrolytiques avec une volonté de comprendre et d'être efficace qui ne pouvait que forcer le respect.

Georges a formé des réanimateurs qui ont fait leurs les principes qu'il défendait. S'intéresser au malade avant tout, savoir être et rester avant tout un bon ouvrier. Il a créé une équipe et nous a inculqué ses valeurs de dévouement, de convivialité et d'humanité. Il disait nous, en parlant de l'équipe du service, et nos travaux, en parlant des articles émanant de l'un d'entre nous.

Viscéralement attaché à l'hôpital public, Georges prenait et exigeait que nous, ses collaborateurs, prenions en charge tous les patients avec la même exigence qu'ils soient atteints d'une pathologie complexe ou au contraire très simple, qu'ils soient très malades ou sur le point de sortir, riches ou pauvres, propres ou sales. Il répétait toujours : «Pas de préjugé, pas de complexe. Tout ce qui brille n'est pas or. Éloignez-vous de la pensée unique ». Personne n'a oublié ses interventions fracassantes lors des congrès où, il posait les questions qui dérangeaient, mais que beaucoup rêvaient en fait de poser.

\section{Défenseur de la discipline}

Georges a été un membre moteur de la Société de réanimation de langue française dont il a été président après avoir été secrétaire général. Il aura assisté à tous les congrès annuels. Personne n'a oublié ses interventions lors du congrès de janvier 2019. Membre du Collège des enseignants de réanimation médicale, membre du CNU de la discipline, coordonnateur du DESC au niveau de l'île-de-France, président du Syndicat national des médecins réanimateurs des hôpitaux publics, Georges a été un défenseur infatigable et visionnaire de la discipline. S'attachant toujours à hausser le niveau des débats, en délaissant les querelles de personnes, ne cherchant qu'à promouvoir l'intérêt général et jamais le sien, il savait poser les véritables questions. On lui doit un traité sur les troubles hydroélectrolytiques et un autre sur les états infectieux graves, mais dans ce domaine sa contribution la plus accomplie est la rédaction et la coordination du Traité de réanimation («le Georges » comme le désigne certains) dont il était en train de finaliser la dernière édition. Je me souviens encore du jour où cette idée lui était venue. «Maury tu sais, il n'y a pas d'ouvrage de référence en français consacré à la réanimation médicale. Il faut en faire un $»$. Et il l'a fait. Ses collaborateurs sur ce projet se souviendront de l'énergie qu'il a pu déployer pour le mener à terme. Enfin, soucieux d'enseigner, de sortir de son service et de développer la réanimation là où elle n'existait pas, il s'est battu pour ouvrir un service de réanimation à l'hôpital du Pakistan Institute of Medical Sciences à Islamabad. Georges avait déployé une force de persuasion peu commune pour mener à bien ce projet rencontrant des administrateurs, des ambassadeurs et même des ministres. Enfin, beaucoup se souviennent de séjours extraordinaires au congrès de la Society of Critical Care Medicine, ou dans des structures prestigieuses comme le NIH ou le CDC où il avait emmené les plus jeunes. On y travaillait avec exigence, mais ensuite la découverte était avec lui un moment extraordinaire.

\section{On ne peut enfin évoquer Georges sans parler du voyageur}

Certains partent en vacances pour se reposer. Je crois que ce concept (se reposer) lui était complètement étranger. Il partait au lever du soleil, déjeunait en marchant ou en conduisant et rentrait à la maison à la nuit tombée. J'ai le souvenir très vif d'un congrès qui se tenait à Santa $\mathrm{Fe}$ où il m'avait emmené pour que j’y présente le résultat de mes travaux. Je le revois, trépignant dans la salle de conférences en bermuda attendant que la discussion de ma communication soit terminée pour m'emmener en disant « On n'est quand même pas venu ici pour faire de la médecine ». Et effectivement pendant les deux jours qui ont suivi nous avons parcouru, pendant $1800 \mathrm{~km}$, le Nouveau-Mexique d'est en ouest du nord au sud suivant les traces des Indiens Navajos et Pueblos. 


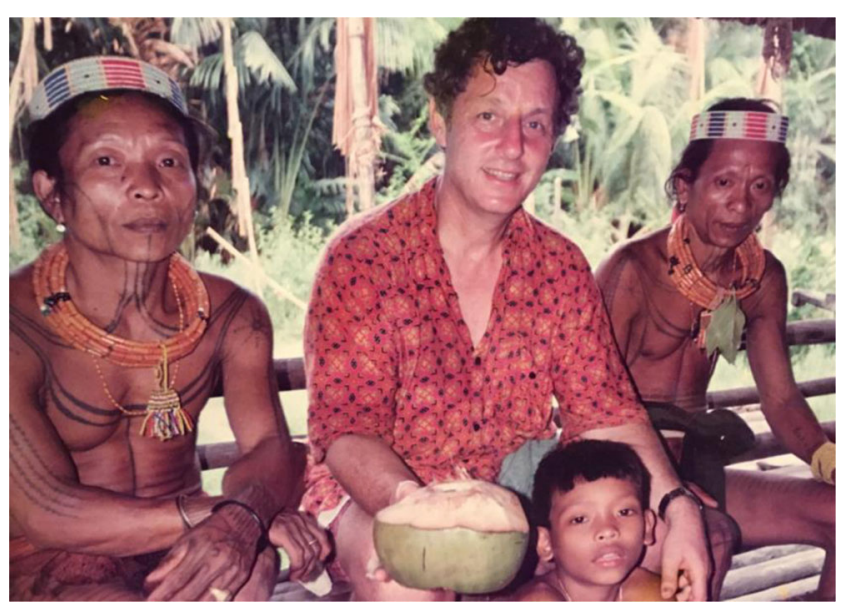

Fig. 2 Indiana Georges
Georges disait avoir besoin d'exotisme. Voir et sentir les fleurs, voir et toucher des animaux, voir la façon dont les gens simples vivent, Zanskar, Papouasie, Pamir, Alaska. Georges a visité ou plutôt exploré les régions les plus inattendues du monde (Fig. 2). C'est en voyageant que la mort l'a brutalement rattrapé.

Nous avons perdu un homme de parole et de conviction, curieux de tout, un défenseur acharné de la discipline, un supporter inconditionnel de la SRLF.

J'ai perdu mon père spirituel.

Nos pensées vont à son épouse Claudine, à ses deux fils Nicolas et Mathieu et à toute sa famille.

$\mathrm{Au}$ revoir Georges! 